\title{
A Historical Account of the Evolution and Emergence of the District Heating Sector in Denmark: How Large-scale Heat Planning Increased Energy Efficiency and Energy Systems Flexibility
}

\author{
Katinka Johansen (DR./MS.) \\ Aalborg University, Copenhagen
}

\begin{abstract}
This historical account discusses the emergence and the evolution of the district heating (DH) sector in Denmark, and it does so using the research methodology of integrative reviewing. DH supplies approximately $65 \%$ of the Danish building mass heating, with some of this heating supplied by the highly energy efficient combined heat and power plants (CHPs). Danish energy policy is characterized by the headlines energy independence, energy efficiency and energy diversification, although underpinned by mixed and interchanging political, economic and environmental rationales throughout the decades. Kick-started in the wake of the oil- and economic crisis in the 70s, this energy policy guided the national scale and long-term energy planning initiatives for the deployment and expansion of DH networks throughout Denmark. This paper focusses on the sociocultural and sociopolitical perspective of this technological journey, and it emphasizes the importance of top down and bottom up initiatives in that regard. The paper argues that while a bottom-up tradition of tinkering, problem solving, pragmatism and innovation has resulted in the technological diversity that characterizes the Danish DH sector today, the top-down policy support and ideology of the emergent welfare state, the cooperative culture and a political tradition of broad coalition agreements were also pivotal for Danish DH sector. Vis-à-vis low-carbon energy transitions, the technical diversity of DH facilitates fuel diversity and energy systems flexibility. This allows for the integration of intermittent and renewable energy in the grid. Lessons learnt and research insights drawn from this historical account are discussed.
\end{abstract}

Keywords: district heating; energy policy; energy transitions; energy efficiency; energy history; 